\title{
A comparative study for the short-term effects of targeted high-intensity UVB and narrow-band UVB in the treatment of vitiligo
}

\author{
MIN ZHANG ${ }^{1}$ and WEI-YUAN MA ${ }^{2}$ \\ ${ }^{1}$ Department of Dermatology, Jinan Central Hospital Affiliated to Shandong University, Jinan, Shandong 250013; \\ ${ }^{2}$ Department of Dermatology, Qilu Hospital of Shandong University, Jinan, Shandong 250012, P.R. China
}

Received March 3, 2015; Accepted January 13, 2017

DOI: $10.3892 /$ etm.2017.4381

\begin{abstract}
Vitiligo is a common acquired depigmentation disorder for which many treatments have previously been used with varying effects. The aim of the present study was to evaluate the efficacy and safety of targeted high-intensity (TH) and narrow band (NB) ultraviolet B light (UVB) for vitiligo treatment. A total of 33 patients with vitiligo were enrolled in the present study. Patients with a symmetrical or near-symmetrical distribution of lesions on their bodies were selected for the present study. For each patient, half of the body was treated with TH-UVB and the other with NB-UVB twice weekly over a period of 12 weeks (24 times total). The patients were assessed each week for repigmentation of lesions. All data were analyzed using SPSS software, and a total of 30 patients were evaluated. The effective rate was significantly higher in the TH-UVB group (56.7\%) compared with the NB-UVB group $(20.0 \%$; $\mathrm{P}<0.05)$. Additionally, the mean number of radiations necessary for initial repigmentation was significantly lower in the TH-UVB group (7.95 \pm 3.43$)$ compared with the NB-UVB group $(15.36 \pm 3.43 ; \mathrm{P}<0.05)$. No correlation was found between the clinical features of patients and the efficacy of treatment. In summary, the results of the present study suggest that TH-UVB is a more effective treatment for vitiligo than NB-UVB.
\end{abstract}

\section{Introduction}

Worldwide, $1-4 \%$ of the population suffers from vitiligo, a commonly acquired depigmentation disorder $(1,2)$. Several etiological factors have been suggested, for which the most compelling evidence involves a combination of environmental,

Correspondence to: Dr Wei-Yuan Ma, Department of Dermatology, Qilu Hospital, Shandong University, 107 West Cultural Road, Jinan, Shandong 250012, P.R. China

E-mail:mwy2015@126.com

Key words: vitiligo, ultraviolet, efficacy genetic and immunological factors interacting to contribute to autoimmune melanocyte destruction (3).

Vitiligo is characterized by round white spots on the skin that grow in size over time or, in rare cases, recover spontaneously (4).

Many treatments are available for patients with vitiligo, with varying degrees of efficacy. Current treatment options include the use of corticosteroids, immunomodulators, skin grafts, pseudocatalase, and phototherapy $(5,6)$. At present, narrow band ultraviolet B light (NB-UVB) is typically used to treat vitiligo due to its satisfactory clinical results (7). However, the phototoxicity of UVB on lesional or non-lesional skins makes high dose delivery dangerous (8). Novel phototherapy devices, such as $308 \mathrm{~nm}$ Xenon Chloride excimer lasers and monochromatic excimer lights (MELs) have emerged that allow phototherapy to be targeted directly to the lesions $(9,10)$. However, these devices are expensive and require a great deal of maintenance (11).

Recently, targeted high intensity (TH)-UVB has been employed for the treatment of localized vitiligo (12). Previous studies have focused on the safety and efficacy of TH-UVB treatment; however, these studies have not included comparisons of different treatments on the same patient (13). In the present study, patients were treated concurrently with TH-UVB and NB-UVB and the results demonstrated that TH-UVB is more effective that NB-UVB for the treatment of vitiligo.

\section{Materials and methods}

Patients. The present study was approved by the Medical Ethics Committee for Human Studies of Shandong University of Medical Sciences (Jinan, China). A total of 33 patients (14 male, 19 female) were selected and gave prior informed consent for the present study. The treatment schedule, including possible benefits and side effects, was clearly explained to all patients. Patients exhibited stable vitiligo with $<10 \%$ skin involvement, and their skin types were type III $(n=2)$ or IV $(n=31)$ on the Fitzpatrick scale (14). Stable vitiligo was defined as clinically unchanged during the past 8 weeks. The required wash-out periods were 4 weeks for all forms of phototherapy and 2 weeks for topical treatments. Patients with a history of skin cancer, psychological disorders, photosensitive conditions 
Table I. Demographic and clinical features of patients $(n=30)$.

\begin{tabular}{|c|c|}
\hline Characteristics & Number $(\%$ \\
\hline \multicolumn{2}{|l|}{ Sex } \\
\hline Male & $14(46.7)$ \\
\hline Female & $16(53.3)$ \\
\hline \multicolumn{2}{|l|}{ Age (years) } \\
\hline $16-20$ & $1(3.3)$ \\
\hline $21-30$ & $9(30.0)$ \\
\hline $31-40$ & $13(43.3)$ \\
\hline $41-50$ & $5(15.7)$ \\
\hline $51-60$ & $2(2.7)$ \\
\hline \multicolumn{2}{|c|}{ Age at onset (years) } \\
\hline $0-10$ & $1(3.3)$ \\
\hline $11-20$ & $4(13.3)$ \\
\hline $21-30$ & $13(43.3)$ \\
\hline $31-40$ & $9(30.0)$ \\
\hline $41-50$ & $3(10.0)$ \\
\hline \multicolumn{2}{|c|}{ Duration of vitiligo (years) } \\
\hline$<1$ & $10(33.3)$ \\
\hline $1-5$ & $7(23.3)$ \\
\hline $6-10$ & $8(26.7)$ \\
\hline$>10$ & $5(16.7)$ \\
\hline \multicolumn{2}{|l|}{ Site } \\
\hline Face/neck & $5(16.7)$ \\
\hline Trunk & $14(46.7)$ \\
\hline Leg/arm & 8 (26.7) \\
\hline Hand/foot & $3(10.0)$ \\
\hline
\end{tabular}

(such as solar urticaria, xeroderma pigmentosum, or chronic actinic dermatitis) or lupus erythematosus, were excluded from the present study, as were those who were lactating or pregnant. Historically, the patients had used treatments including topical steroids, topical calcipotriol, or Chinese traditional medicines with variable success. Patients had a mean age of $34.3 \pm 8.9$ years, and had suffered from vitiligo for $34.3 \pm 8.9$ months. Patient demographics and clinical features are provided in Table I.

Study lesions. A total of 32 symmetrically-distributed or near-symmetrically-distributed pairs of vitiligo patches were selected for testing. The body of the patient was divided into treatment halves symmetrically along the sagittal plane. Lesions on one side of the body were treated with TH-UVB, whereas those on the other side were treated with NB-UVB.

Phototherapy device. A Dua-Light UV120-2 TH-UVB device (Theralight, Inc., Carlsbad, CA, USA) with a high-pressure mercury lamp was used to administer TV-UVB treatment. The light source is able to output UVB spectral peaks at 302 and $312 \mathrm{~nm}$ wavelengths, and the mean weighted erythemal wavelength was $304 \mathrm{~nm}$ and is able to administer a high output of UVB irradiation of $100 \mathrm{~mJ} / \mathrm{cm}^{2}$ in $\sim 0.7 \mathrm{sec}$. The UV radiation is delivered through a $1.9 \times 1.9 \mathrm{~cm}$ square aperture.
A UV109B NB-UVB device (Herbert Waldmann GmbH and Co., KG, Villingen-Schwenningen, Germany) was used for NB-UVB treatment, containing 48 fluorescent tubes with peak emissions at $311-312 \mathrm{~nm}$.

Interventions. The minimal erythema dose (MED) was established for each patient in the TH-UVB group. The Dua-Light system is equipped with an MED determination function with set increasing doses of light for each skin type. The first administered dose was 2MED, and the subsequent dosing plan was as follows: $10 \%$ increase if no erythema was induced by prior irradiations, $5 \%$ increase if erythema lasted for $<24 \mathrm{~h}$, or no increase if erythema lasted $>24 \mathrm{~h}$. If pain or blistering occurred, treatment was put on hold until these symptoms had resolved and the dose was decreased by $10 \%$. For the NB-UVB treatment, the first dose was 70\% MED. The subsequent dose was increased by $10 \%$ if no erythema occurred or erythema lasted for $<24 \mathrm{~h}$, or kept the same if erythema lasted for 24-72 h. If pain or blistering developed or erythema lasted $>72 \mathrm{~h}$, treatment was suspended until these symptoms resolved, and the dose was reduced by $10 \%$.

Evaluation of efficacy. To evaluate repigmentation, two investigators assessed the lesions based on the percentage of repigmentation of the total vitiliginous area in each patient through digital image projections as follows: Excellent $(>75 \%)$, good $(50-75 \%)$, moderate $(25-50 \%)$, and mild $(<25 \%)$. The effective rate was calculated as a percentage as follows: [nx(excellent+good+moderate)/n(total) $] \times 100$.

Statistical analysis. The enumeration data was analyzed using Chi-square. The measurement data were analyzed using Student's t-test. Independent prognostic factors were determined by the multivariate analysis through binary logistic regression. $\mathrm{P}<0.05$ was considered to indicate a statistically significant difference. The SPSS software package (version 13.0; SPSS, Inc., Chicago, IL, USA) was used for all statistical analyses.

\section{Results}

Clinical features of patients. Throughout the course of the present study, 3 patients dropped out. The 30 patients (14 male, 16 female) who successfully completed the study were used for analysis. At the end of the study, patients had received a total of 24 treatments with TH-UVB and NB-UVB on the appropriate side (Table I).

Efficacy of TH-UVB and NB-UVB. Fig. 1 shows representative lesions for each treatment group. Total repigmentation was not observed in any of the evaluated areas (Table II). Following 8 treatments, 5 (16.7\%) lesions treated with TH-UVB and one (3.3\%) lesion treated with NB-UVB exhibited moderate repigmentation, with no significant differences observed. Following 16 treatments, $2(6.7 \%)$ lesions treated with TH-UVB displayed good repigmentation and $15(50.0 \%)$ had moderate repigmentation, whereas only $5(16.7 \%)$ lesions treated with NB-UVB exhibited moderate repigmentation. The effective rate of TH-UVB was significantly greater than that of NB-UVB $(\mathrm{P}<0.05)$.

Following 24 treatments, the repigmentation of $5(16.7 \%)$ and $12(40.0 \%)$ TH-UVB-treated lesions was considered to 
Table II. Efficacy of TH-UVB and NB-UVB.

\begin{tabular}{|c|c|c|c|c|c|c|}
\hline No. of treatments & Group & Mild (n) & Moderate (n) & Good (n) & Excellent (n) & Effective rate $(\%)$ \\
\hline 4 & TH-UVB & 29 & 1 & 0 & 0 & 3.3 \\
\hline 4 & NB-UVB & 30 & 0 & 0 & 0 & 0.0 \\
\hline 8 & TH-UVB & 25 & 5 & 0 & 0 & 16.7 \\
\hline 8 & NB-UVB & 29 & 1 & 0 & 0 & 3.3 \\
\hline 16 & TH-UVB & 13 & 15 & 2 & 0 & 56.7 \\
\hline 16 & NB-UVB & 25 & 5 & 0 & 0 & 16.7 \\
\hline 24 & TH-UVB & 13 & 12 & 5 & 0 & 56.7 \\
\hline 24 & NB-UVB & 24 & 5 & 1 & 0 & 20.0 \\
\hline
\end{tabular}

TH, targeted high-intensity; UVB, ultraviolet b light; NB, narrow band; mild, <25\% repigmentation; moderate, 25-50\% repigmentation; good, $50-75 \%$ repigmentation; excellent, $>75 \%$ repigmentation.

Table III. The effective rates of TH-UVB and NB-UVB at various sites.

\begin{tabular}{lcc}
\hline Site & $\begin{array}{c}\text { TH-UVB } \\
\text { Effective rate (\%) }\end{array}$ & $\begin{array}{c}\text { NB-UVB } \\
\text { Effective rate (\%) }\end{array}$ \\
\hline Face/neck & 40.0 & 0.0 \\
Trunk & 85.7 & 42.9 \\
Leg/arm & 0.0 & 0.0 \\
Hand/feet & 0.0 & 0.0 \\
\hline
\end{tabular}

TH, targeted high-intensity; UVB, ultraviolet b light; NB, narrow band.

be good and moderate, respectively. In contrast, only $1(3.3 \%)$ NB-UVB-treated lesion exhibited good repigmentation and $5(16.7 \%)$ exhibited moderate repigmentation. The effective rates in TH-UVB and NB-UVB treated areas were calculated to be 56.7 and $20 \%$, respectively. These results demonstrate that the effective rate of TH-UVB was significantly greater than that of NB-UVB $(\mathrm{P}<0.05)$.

The earliest repigmentation with TH-UVB treatment was observed following 3 treatments, and the mean number of treatments required for initial repigmentation was 7.95 \pm 3.43 . However, for lesions treated with NB-UVB, the earliest appearance of repigmentation was observed following 10 treatments, and the mean number of treatments required for initial repigmentation was $15.36 \pm 3.43$. The mean number of treatments required for initial repigmentation was significantly lower for TH-UVB treatment compared with NB-UVB treatment $(\mathrm{P}<0.05)$.

Effective rates of $T H-U V B$ and $N B-U V B$ at various sites. The effective rates at different sites are presented in Table III. The effective rates of improvement of lesions on the trunk were higher than that of lesions on the face and neck both in TH-UVB and NB-UVB group $(\mathrm{P}<0.05)$. None of the lesions on the hands, feet, legs or arms were considered to show even moderate improvement. No correlation was found between efficacy of treatment and age, sex, age at onset, duration of
A
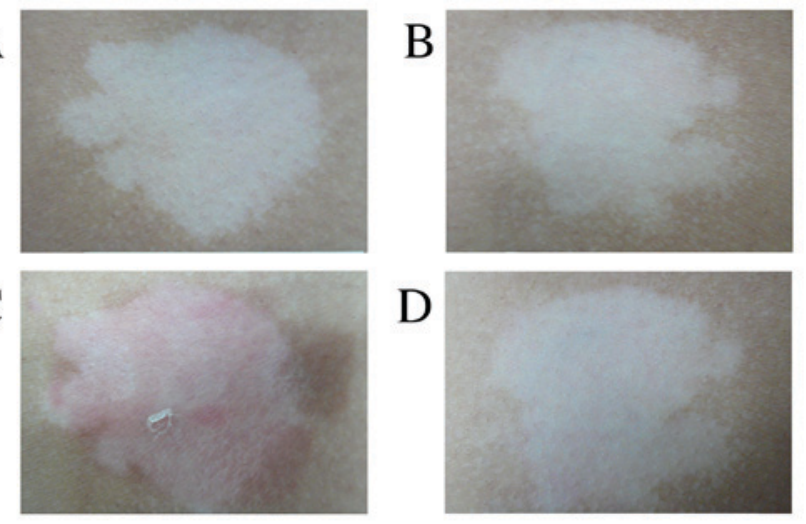

$\mathrm{E}$
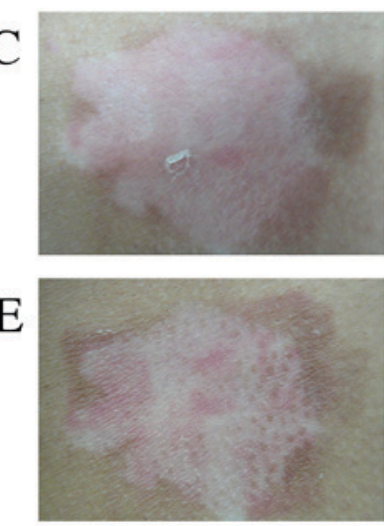

$\mathrm{F}$

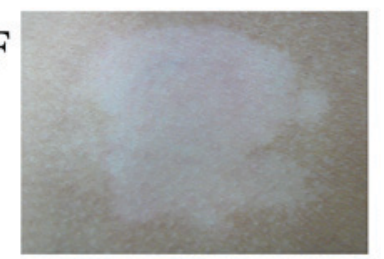

G

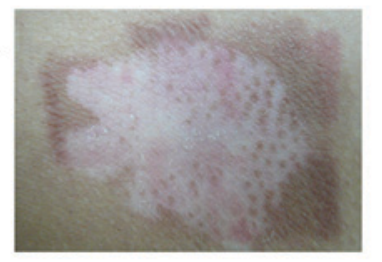

$\mathrm{H}$

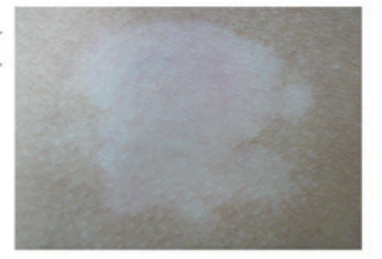

Figure 1. Vitiligo lesions on the trunk of a male 46-year-old patient. Images are from before treatment with (A) TH-UVB or (B) NB-UVB, after 2 treatments with (C) TH-UVB or (D) NB-UVB, after 6 treatments with (E) TH-UVB or (F) NB-UVB and after 12 treatments with (G) TH-UVB or (H) NB-UVB. TH-UVB, TH, targeted high-intensity; UVB, ultraviolet b light; NB, narrow band.

vitiligo or site of lesions. Only mild adverse events, such as scaling and itching, were observed, and these symptoms were relieved by the application of moisturizer.

\section{Discussion}

Vitiligo treatment has historically been a therapeutic challenge. Phototherapy is considered to be one of the most effective therapeutic treatment methods $(5,6)$. For example, NB-UVB, 
the efficacy of which has been demonstrated in various patient populations, has become an established treatment (7). NB-UVB is the preferred method of treatment, particularly in patients with active and generalized vitiligo, with an effectiveness of $63 \%$ against this form of the disease (15). NB-UVB has previously been demonstrated to be highly effective in the treatment of stable vitiligo when $\geq 5 \%$ of the body is affected (16). This therapy is very safe and is suitable to be administered to children (17).

Several targeted phototherapy devices have been tested in clinical trials, such as $308 \mathrm{~nm}$ Xeon-Chloride excimer lasers, non-laser $308 \mathrm{~nm}$ monochromatic excimer lights, and TH-UVB (18). These devices have been demonstrated to offer some therapeutic advantages, such as a rapid onset of repigmentation and fewer treatments needed to achieve satisfactory repigmentation than the conventional NB-UVB. Asawanonda et al (19) used 12-week TH-UVB phototherapy to treat 29 vitiliginous lesions in 6 patients, and repigmentation occurred in all cases to varying degrees. However, the responses varied between the different anatomic locations, with the least improvement observed in acral lesions. A 50-75\% repigmentation was observed in one lesion and $>25 \%$ was observed in 17 lesions. It was concluded that this device was only efficacious for the treatment of localized vitiligo. Akar et al (20) used TH-UVB to treat 32 patients with localized vitiligo. Following 20-60 treatment sessions, visible repigmentation was observed in only 4 patients. Two patients exhibited $>75 \%$ repigmentation, whereas the other two patients exhibited mild repigmentation. Facial lesions were the only lesions shown to be responsive to the treatment. It was concluded that the clinical effectiveness of TH-UVB was limited and determined by the location of vitiligo lesions.

In the present study, a greater efficacy and earlier onset of repigmentation was observed in lesions treated with TH-UVB compared with NB-UVB in the majority of patients. These results indicate that the effective rate is higher for TH-UVB-treated patches than for NB-UVB-treated patches; however, it is unclear whether TH-UVB remains more effective than NB-UVB phototherapy following 24 irradiation treatments.

TH-UVB treatment is able to limit exposure to radiation by targeting only selected areas of skin, and the results of the present study demonstrate that positive results are achieved very quickly with better efficacy than with NB-UVB phototherapy. Furthermore, patient motivation and compliance may be enhanced due to the rapid onset of repigmentation. The therapeutic effects of the two UVB treatments may be similar as, photobiologically, their wavelengths (TH-UVB, $304 \mathrm{~nm}$; NB-UVB, $311 \mathrm{~nm}$ ) are close to each other. The underlying mechanism for the improvement of vitiligo with UVB treatment remains to be elucidated. Some authors have suggested that the cytotoxic effect of UVB on infiltrating T cells, where the mechanism of cell death is most likely apoptosis, is the major mechanism (21). The majority of authors believe that the capacity of UVB irradiation to induce T cell apoptosis is associated with its clinical efficacy $(22,23)$. Future studies investigating whether TH-UVB is more effective at inducing $\mathrm{T}$ cell apoptosis than NB-UVB may help to elucidate the underlying mechanisms.

In conclusion, the results of the present study demonstrate that TH-UVB is a more effective phototherapy than
NB-UVB in the treatment of vitiligo. TH-UVB also has distinct advantages as it may be used in hard-to-reach areas to treat focal lesions. Furthermore, higher doses of TH-UVB may be centrally delivered without exposing unaffected skin to radiation. However, the findings of the present study should be confirmed and extended with long-term studies. Future studies with larger sample sizes and number of treatments should be employed to further demonstrate the clinical effects of UVB.

\section{Acknowledgements}

The authors of the present study would like to thank Shandong University for its cooperation.

\section{References}

1. Schallreuter KU, Levenig C and Berger J: Vitiligo and cutaneous melanoma. A case study. Dermatologica 183: 239-245, 1991.

2. Czajkowski R and Męcińska-Jundziłł K: Current aspects of vitiligo genetics. Postepy Dermatol Alergol 31: 247-255, 2014.

3. Allam M and Riad H: Concise review of recent studies in vitiligo. Qatar Med J 2013: 1-19, 2013.

4. Le Poole IC, Das PK, van den Wijngaard RM, Bos JD and Westerhof W: Review of the etiopathomechanism of vitiligo: A convergence theory. Exp Dermatol 2: 145-153, 1993.

5. Ezzedine K, Eleftheriadou V, Whitton M and van Geel N: Vitiligo. Lancet 386: 74-84, 2015.

6. Ghafourian A, Ghafourian S, Sadeghifard N, Mohebi R, Shokoohini Y, Nezamoleslami S and Hamat RA: Vitiligo: Symptoms, pathogenesis and treatment. Int J Immunopathol Pharmacol 27: 485-489, 2014.

7. Linthorst Homan MW, Spuls PI, Nieuweboer-Krobotova L, de Korte J, Sprangers MA, Bos JD, Wolkerstorfer A and van der Veen JP: A randomized comparison of excimer laser versus narrow-band ultraviolet $\mathrm{B}$ phototherapy after punch grafting in stable vitiligo patients. J Eur Acad Dermatol Venereol 26: 690-695, 2012.

8. Lotti T, Berti S and Moretti S: Vitiligo therapy. Expert Opin Pharmacother 10: 2779-2785, 2009.

9. Greve B, Raulin C and Fischer E: Excimer laser treatment of vitiligo-critical retrospective assessment of own results and literature overview. J Dtsch Dermatol Ges 4: 32-40, 2006 (In German).

10. Verhaeghe E, Lodewick E, van Geel $\mathrm{N}$ and Lambert J: Intrapatient comparison of 308-nm monochromatic excimer light and localized narrow-band UVB phototherapy in the treatment of vitiligo: A randomized controlled trial. Dermatology 223: 343-348, 2011.

11. Lopes C, Trevisani VF and Melnik T: Efficacy and safety of 308-nm monochromatic excimer lamp versus other phototherapy devices for vitiligo: A systematic review with meta-analysis. Am J Clin Dermatol 17: 23-32, 2016.

12. Welsh O, Herz-Ruelas ME, Gómez M and Ocampo-Candiani J: Therapeutic evaluation of UVB-targeted phototherapy in vitiligo that effects less than $10 \%$ of the body suiface area. Int J Dermatol 48: 529-534, 2009.

13. Asawanonda P, Charoenlap M and Korkij W: Treatment of localized vitiligo with targeted broadband UVB phototherapy: A pilot study. Photodermatol Photoimmunol Photomed 22: 133-136, 2006.

14. Fitzpatrick TB: The validity and practicality of sun-reactive skin types I through VI. Arch Dermatol 124: 869-871, 1988.

15. Njoo MD, Westerhof W, Bos JD and Bossuyt PM: The development of guidelines for the treatment of vitiligo. Clinical Epidemiology Unit of the Istituto Dermopatico dell'Immacolata-Istituto di Recovero e Cura a Carattere Scientifico (IDI-IRCCS) and the Archives of Dermatology. Arch Dermatol 135: 1514-1521, 1999.

16. Hamzavi I, Jain H, McLean D, Shapiro J, Zeng H and Lui H: Parametric modeling of narrowband UV-B phototherapy for vitiligo using a novel quantitative tool: The Vitiligo Area Scoring Index. Arch Dermatol 140: 677-683, 2004.

17. Darné S, Leech SN and Taylor AE: Narrowband ultraviolet $\mathrm{B}$ phototherapy in children with moderate-to-severe eczema: A comparative cohort study. Br J Dermatol 170: 150-156, 2014. 
18. Roelandts R: Photo(chemo)therapy for vitiligo. Photodermatol Photoimmunol Photomed 19: 1-4, 2003.

19. Asawanonda P, Charoenlap M and Korkij W: Treatment of localized vitiligo with targeted broadband UVB phototherapy: A pilot study. Photodermatol Photoimmunol Photomed 22: 133-136, 2006.

20. Akar A, Tunca M, Koc E and Kurumlu Z: Broadband targeted UVB phototherapy for localized vitiligo: A retrospective study. Photodermatol Photoimmunol Photomed 25: 161-163, 2009.

21. Hashimoto A, Sato T, Iyama S, Yoshida M, Ibata S, Tatekoshi A Kamihara Y, Horiguchi $\mathrm{H}$, Murase $\mathrm{K}$, Kawano Y, et al: Narrow-band ultraviolet B phototherapy ameliorates acute graft-versus-host disease of the intestine by expansion of regulatory T cells. PLoS One 11: e0152823, 2016.
22. Ozawa M, Ferenczi K, Kikuchi T, Cardinale I, Austin LM, Coven TR, Burack LH and Krueger JG: 312-nanometer ultraviolet $\mathrm{B}$ light (narrow-band UVB) induces apoptosis of T cells within psoriatic lesions. J Exp Med 189: 711-718, 1999.

23. Bianchi B, Campolmi P, Mavilia L, Danesi A, Rossi R and Cappugi P: Monochromatic excimer light (308 nm): An immunohistochemical study of cutaneous $\mathrm{T}$ cells and apoptosis-related molecules in psoriasis. J Eur Acad Dermatol Venereol 17: 408-413, 2003. 